\title{
Photophysical Behaviour of Novel Quaternary Pyrenoyl Salts and Their Sensitivity Towards Bile Salt Micellization
}

\section{Saravanan}

B S Abdur Rahman Crescent Institute of Science \& Technology

S. Dhivakar

B S Abdur Rahman Crescent Institute of Science \& Technology

M. Mohapatra ( mmohapatra@gmuniversity.ac.in )

Gangadhar Meher University https://orcid.org/0000-0003-0972-2037

S. Kutti Rani

B S Abdur Rahman Crescent Institute of Science \& Technology

K. Vijayakumaran

Polyvalent Organics

\section{Research Article}

Keywords: Pyrenoyl salts, Bile salt, Micellization, Sodium deoxycholate, Fluorescence

Posted Date: February 7th, 2022

DOI: https://doi.org/10.21203/rs.3.rs-1308526/v1

License: (9) This work is licensed under a Creative Commons Attribution 4.0 International License. Read Full License 


\section{Abstract}

The present work describes the synthesis and characterization of pyrene derivatives, $\mathrm{N}-(1-$ Pyrenoylmethyl)pyridinium bromide (PM-PB) and N-(1-Pyrenoylmethyl)-N,N,N-triethylammonium bromide (PM-TAB). Photophysical behavior of these molecules has been studied in various protic and aprotic solvents. Using steady state fluorescence intensity, fluorescence anisotropy and dynamic fluorescence lifetime studies, the sensitivity of these molecules towards the micellization process of bile salts has been monitored. These derivatives have been effectively used in estimating critical micellar concentration (CMC) of bile salt, sodium deoxycholate (NaDC).

\section{Introduction}

Pyrene is an interesting fluorescent molecule for preparing organic electronic materials. ${ }^{1}$ Over the past decade, many pyrene-based dyes have been synthesized, and their photophysical properties have been studied [1-4]. Fluorescence of pyrene derivatives always has potential application in studying organized and microheterogeneous media [3, 4]. The photophysical properties of pyrenecarbonyl compounds like 1methoxycarbonylpyrene, 1-acetylpyrene, pyrene-3-carboxaldehyde, 1-pyrenecarboxylic acid, are well known and have widely been used in various fields [1]. Polarity sensitive, sparingly soluble fluorophore pyrene-3-carboxaldehyde and $\mathrm{pH}$ sensitive 1-Pyrenecarboxylic acid makes them useful derivatives of pyrene $[1,5-7]$. The understanding of photophysical properties of carbonyl functionalized pyrenes is essential for material design and for expanding the scope of their applications.

Physiological importance of bile salts lies in their potentiality to solubilize fat soluble vitamins, cholesterol, and lipids in the hepatobiliary ststems [8-10]. These biosurfactants have obtained much recognition due to their application in delivery systems for medicines, cosmetics etc. Bile salts at their lower concentrations are known to hydrate the lipid bilayer membranes [11-13]. Micelle forming ability of bile salts is due to the presence of hydrophobic and hydrophilic parts in the same molecule. Micellization process brings about several changes in a surfactant solution. This results in appreciable alteration in surface tension, viscosity, light scattering phenomenon etc., which makes the basis of several analytical techniques for the investigation of micelle formation [14-18]. For monitoring biological activities of bile salts the critical micelle concentration (CMC) is an important fundamental parameter. Over the years, fluorescence spectroscopy has been used as an important technique to study the CMC of bile salts [4, 18-21].

The main objectives of the present work are (i) synthesis and characterization of the carbonyl functionalized pyrene derivatives, $\mathrm{N}$-(1-Pyrenoylmethyl)pyridinium bromide (PM-PB) and N-(1Pyrenoylmethyl)-N,N,N-triethylammonium bromide (PM-TAB); (ii) detailed photophysical investigations of the synthesized molecules in homogeneous media; (iii) sensitivity of these molecules towards micellization process of bile salt, sodium deoxycholate $(\mathrm{NaDC})$.

\section{Materials And Methods}




\subsection{Materials}

$\mathrm{N}$-(1-Pyrenoylmethyl)pyridinium bromide (PM-PB) and N-(1-Pyrenoylmethyl)-N,N,N-triethylammonium (PM-TAB) were synthesized and purified by repeated recrystrallization Reagent grade chemicals were used and solvents were purified by standard techniques. Melting points were determined using Vego digital programmable melting point device. Thin Layer Chromatography (TLC) was carried out using Merck aluminum TLC plates, silica gel coated with flourescent indicator F254. HPLC analysis was performed using Waters Alliance 2695 instrument with PDA 2998 detector. Bile salt; sodium deoxycholate $(\mathrm{NaDC})$ was obtained from S.D. Fine Chemicals Ltd. For the photophysical study all solvents used were of spectroscopic grade. Triple distilled water, purified using $\mathrm{KMnO}_{4}$ and $\mathrm{NaOH}$, was used for the preparation of bile salt solution.

\subsection{Sample preparation for photophysical studies}

The stock solutions $\left(10^{-3} \mathrm{M}\right)$ of pyrene derivatives were prepared in methanol. The solutions in all other solvents were prepared by evaporating the methanol from the desired amount of the stock by gentle purging of nitrogen gas and then sonicating it with the solvent of interest. A set of NaDC solutions in the concentrations range of $1-18 \mathrm{mM}$ containing the same amount of pyrene derivatives $\left(5 \times 10^{-5} \mathrm{M}\right)$, was prepared in water at neutral $\mathrm{pH}$. The synthesized compounds are quite insoluble in water. All these experiments were carried out at room temperature.

\subsection{Absorption and steady state fluorescence experiments}

Absorption spectra were collected using a Jasco V-650 UV-Vis spectrometer. Steady state fluorescence intensity and fluorescence anisotropy measurements were carried out using a Horiba Jobin-Yvon FluoroMax-4 spectrofluorimeter, with a $150 \mathrm{~W}$ xenon lamp as the light source. The emission spectra and fluorescence anisotropy were recorded with slit widths of $3 / 3 \mathrm{~nm}$. The excitation wavelength was fixed at $370 \mathrm{~nm}$.

\subsection{Fluorescence lifetime measurements}

Fluorescence decay dynamic studies were carried out using Horiba Jobin Yvon TCSPC lifetime instrument in a time-correlated, single photon counting arrangement. $370 \mathrm{~nm}$ nano-LED was used as the light source. The instrument response function was collected using a scatterer (Ludox AS40 colloidal silica). The decay data were analyzed using IBH software. A value of $\chi 2$ of $0.99 \geq \chi 2 \leq 1.3$ was considered as a good fit which was further confirmed by the symmetrical distribution of the residuals.

\section{Results And Discussion}

\subsection{Synthesis and Characterization of Pyrene derivatives}

\subsubsection{1-Acetylpyrene [22]}


To nitrobenzene (redistilled, $700 \mathrm{ml}$ ), aluminium chloride (anhydrous, $140 \mathrm{~g}, 1.05$ mole), acetic anhydride (50 ml. 0.53 mole) were added under stirring and cooled to $10 \mathrm{deg}$ C. Then, pyrene (recrystallized from acetone, $100 \mathrm{~g}, 0.49$ mole) was added over a period of 30 minutes, under stirring. After 6 hours the reaction was complete and the contents were poured into ice water $(2 \times 150 \mathrm{ml})$ and conc. $\mathrm{HCl}(70 \mathrm{ml})$ and layers separated. The reaction was monitored by Thin layer chromatography (TLC). Nitrobenzene was removed by steam distillation The green-coloured crude product $(140 \mathrm{~g})$ was recrystallized from hot isopropanol.

Yield: $85 \mathrm{~g}$ (yellow crystals, 71\%).

MP: 87-89 deg C (86-89 deg C) [22]

\subsubsection{1-(Bromoacetyl)pyrene [23-25]}

1-Acetylpyrene (10.0 g, $0.04 \mathrm{~m}$ ) was dissolved in tetrahydrofuran(THF) (redistilled and dried, $250 \mathrm{ml}$ ). Then phenyltrimethylammonium tribromide $(16.0 \mathrm{~g}, 0.043 \mathrm{~m})$ was added under magnetic stirring for 3 hours at ambient temperature. The reaction was monitored by TLC. The crystalline precipitate (phenyltrimethylammonium bromide) was filtered and the solvent was concentrated under reduced pressure. The orange coloured crude product $(16.0 \mathrm{~g})$ was recrystallised from hot benzene.

Yield: $11.2 \mathrm{~g}$ (yellow crystals, 85.5\%).

MP. 133-135 deg C (128-129 deg C) [26]

\subsubsection{N-(1-Pyrenoylmethyl)pyridinium bromide [27]}

To acetonitile ( $25 \mathrm{ml}$, reagent grade) were added 1-(bromoacetyl)pyrene $(5.0 \mathrm{~g}, 0.015 \mathrm{~m})$ and pyridine (redistilled, $1.3 \mathrm{ml}, 0.016 \mathrm{~m}$ ) and heated to reflux for 6 hours in an oil bath and the reaction monitored by TLC. The yellow solid formed was filtered and washed with hot benzene to afford the crude yellow powder $(6.0 \mathrm{~g})$ which was recrystallised from redistilled N,N-Dimethylformamide.

Yield: $5.1 \mathrm{~g}$ (yellow powder, 84.5.\%).

MP: 276-279 deg C (decomp.); purity: 99.98\% (High performance liquid chromatography (HPLC))

\subsubsection{N-(1-Pyrenoylmethyl)-N,N,N-triethylammonium bromide [28]}

To acetonitile (50 ml, reagent grade) were added 1-(bromoacetyl)pyrene $(2.0 \mathrm{~g}, 0.006 \mathrm{~m})$ and triethylamine (redistilled, $1.5 \mathrm{ml}, 0.011 \mathrm{~m}$ ) and heated to reflux for 6 hours in an oil bath and the reaction monitored by TLC. The yellow solid formed was filtered and washed with hot benzene to afford the crude yellow powder $(2 \mathrm{~g})$ which was recrystallised from redistilled acetonitrile.

Yield: $1.6 \mathrm{~g}$ (orange powder, 62.8\%). 
MP: 217-219 deg C (decomp.); purity: 97.74\% (HPLC)

The synthesized pyrene derivatives N-(1-Pyrenoylmethyl)pyridinium bromide (PM-PB) and N(Pyrenoylmethyl)triethyl ammonium bromide (PM-TAB) are shown in Fig. 1.

\subsection{Photophysical studies of PM-PB and PM-TAB in homogeneous media}

\subsubsection{Absorption Studies:}

Figure $2 \mathrm{~A}$ and $\mathrm{B}$ represents the UV-Vis absorption spectra of PM-PB and PM-TAB in solvents of varying polarity, respectively. The absorption spectra of both the molecules extended well into the visible region. The absorption spectra of $\mathrm{N}$-(1-Pyrenoylmethyl) pyridinium bromide (PM-PB) do not change much with solvents of different polarity. This indicates within the short time scale $\left(10^{-15} \mathrm{~s}\right)$ of the absorption phenomenon, $\mathrm{PM}-\mathrm{PB}$ is insensitive to the solvent microenvironment around it. In the same set of solvents, the absorption spectrum of PM-TAB was also recorded. It has been observed that on changing solvent polarity PM-TAB shows almost similar absorption features as that of PM-PB. In both the case there is not much change in the energy of transition in different solvents, which implies that stabilization of the ground state species of the compounds by the solvents is not significant.

\subsubsection{Steady State Fluorescence Studies:}

Figure $3 A$ and $B$ shows the normalized steady state fluorescence spectra of PM-PB and PM-TAB in various solvents of different polarity, respectively. Fig. $\mathrm{S} 1$ in the supporting information shows the emission spectra of PM-PB and PM-TAB without normalization indifferent solvents. With increase in solvent polarity from heptane to water, there is a negligible red shift of $\sim 10 \mathrm{~nm}$ in the spectral profile of PM-PB. In non-interacting solvents (like heptane and cyclohexane) the emission spectra of PM-PB and PM-TAB are more structured as compared to that in other solvents of moderate and high polarity. However, PM-TAB shows a remarkable red shift of $\sim 50 \mathrm{~nm}$ in its emission spectral profile with increase in solvent polarity. Both the molecules are not completely soluble in water and showed a very weak fluorescence (Fig. S1 in the supporting information).

The Stokes shift of PM-PB and PM-TAB fits well with ET(30) scales of solvent polarity (Fig. 4) [2, 29]. ET (30) scale is the popularly used solvatochromic scale in the literature. It represents the transition energy in $\mathrm{kcal} \mathrm{mol}^{-1}$ for the longest wavelength absorption band of the dissolved pyridinium-N-phenoxide betaine dye. Correlation of solvent-dependent properties with the ET(30) scale often follows two distinct lines, one for the aprotic and another for the protic solvents. In the present case, ET (30) plot for PM-PB and PMTAB shows two different slopes corresponding to aprotic and protic solvents.

\subsection{Interaction of PM-PB and PM-TAB with bile salt ( $\mathrm{NaDC})$ 3.3.1. Steady State Fluorescence Intensity and Anisotropy Studies:}


The variation of fluorescence intensity of PM-PB and PM-TAB with concentration of NaDC is shown in Fig. $\mathrm{S} 2$ in the supporting information. The corresponding plot of fluorescence intensity of PM-PB and PMTAB with NaDC concentration has been given in Fig. $5 \mathrm{~A}$ and $\mathrm{B}$, respectively. With the concentration of $\mathrm{NaDC}$, fluorescence intensity of both the molecules shows a sigmoid variation. The $\mathrm{CMC}$ point has been represented by the point of intersection of the horizontal line and the line of inflation (shown as dashed lines in Fig. $5 \mathrm{~A}$ and $\mathrm{B}$ ). It is found to be $5 \mathrm{mM}$ for $\mathrm{NaDC}$. This is in agreement with the reported values range from 2-10mM for $\mathrm{NaDC}[4,18-21]$. With increase in NaDC concentration, a remarkable increase in the fluorescence intensity of PM-PB and PM-TAB suggests that hydrophobic bile salt micellar aggregates provides a solubilizing environment to the molecules.

In an organized media, the fluorescence anisotropy of a fluorescent molecule indicates the resistance offered by the microenvironment to its rotational movement. Hence, the change in the polarization of PM$\mathrm{PB}$ and $\mathrm{PM}-\mathrm{TAB}$ fluorescence acts as an index for the change in microviscosity of the immediate environment $[3,4,13]$. Fig. $6 A$ and $B$ represents the plots for the variation of steady state fluorescence anisotropy of PM-PB and PM-TAB as a function of NaDC concentration. Increase in fluorescence anisotropy with increasing NaDC concentration indicates restricted rotational mobility of PM-PB and PMTAB in the progressive bile salt micellar environment.

\subsubsection{Fluorescence Lifetime Studies:}

Time resolved fluorescence decay studies have been performed to have a better understanding of the sensitivity of PM-PB and PM-TAB towards micellar aggregation of NaDC. Both the molecules show biexponential decay profile in presence and absence of $\mathrm{NaDC}$ (Fig. 7). The lifetime values of PM-PB and PM-TAB with increasing NaDC concentration has been given in Table 1. Unlike pyrene, there molecules show short lifetime values.

Table 1. Fluorescence lifetime values of(A) PM-PB and (B) PM-TAB with increasing NaDC concentration from 0to $18 \mathrm{mM}$. $\left(\lambda_{\mathrm{ex}}=370 \mathrm{~nm}, \lambda_{\mathrm{em}}=450 \mathrm{~nm}\right)$ 


\begin{tabular}{|c|c|c|c|c|}
\hline$[\mathrm{NaDC}] \quad(\mathrm{mM})$ & $t_{s}(n s),\left(a_{1}\right)$ & $t_{1}(n s),\left(a_{2}\right)$ & $\mathrm{t}_{\mathrm{avg}}(\mathrm{ns})$ & $c^{2}$ \\
\hline 0 & $0.14(25)$ & $5.29(75)$ & 5.24 & 1.18 \\
\hline 1 & $0.17(23)$ & $5.41(77)$ & 5.36 & 1.10 \\
\hline 3 & $0.81(28)$ & $5.13(72)$ & 4.88 & 1.43 \\
\hline 4 & $1.02(54)$ & $5.20(46)$ & 4.42 & 1.41 \\
\hline 5 & $1.50(87)$ & $4.84(13)$ & 2.59 & 1.36 \\
\hline 6 & $1.56(90)$ & $4.88(10)$ & 2.42 & 1.22 \\
\hline 7 & $1.60(90)$ & $4.78(10)$ & 2.39 & 1.24 \\
\hline 8 & $1.62(91)$ & $5.07(09)$ & 2.43 & 1.24 \\
\hline 10 & $1.64(90)$ & $4.73(10)$ & 2.39 & 1.32 \\
\hline 14 & $1.62(88)$ & $4.30(12)$ & 2.33 & 1.38 \\
\hline 18 & 1.61 (87) & $4.14(13)$ & 2.31 & 1.34 \\
\hline
\end{tabular}

(B) 


\begin{tabular}{|c|c|c|c|c|}
\hline$[\mathrm{NaDC}] \quad(\mathrm{mM})$ & $\mathrm{t}_{\mathrm{s}}(\mathrm{ns}),\left(\mathrm{a}_{1}\right)$ & $t_{1}(n s),\left(a_{2}\right)$ & $t_{\text {avg }}(n s)$ & $c^{2}$ \\
\hline 0 & $0.68(57)$ & $4.12(43)$ & 3.50 & 1.15 \\
\hline 1 & $0.69(59)$ & $4.04(41)$ & 3.38 & 1.23 \\
\hline 3 & $0.69(57)$ & $3.86(43)$ & 3.25 & 1.12 \\
\hline \multirow[t]{2}{*}{4} & $0.70(62)$ & 3.91 & 3.18 & 1.41 \\
\hline & & (38) & & \\
\hline 5 & $0.71(78)$ & 3.01 (22) & 1.96 & 1.15 \\
\hline 6 & $0.74(76)$ & $2.93(24)$ & 1.96 & 1.14 \\
\hline \multirow[t]{2}{*}{7} & $0.78(83)$ & 2.95 & 1.73 & 0.97 \\
\hline & & (17) & & \\
\hline \multirow[t]{2}{*}{8} & $0.84(81)$ & 2.86 & 1.72 & 0.95 \\
\hline & & (19) & & \\
\hline 10 & $0.95(79)$ & $2.74(21)$ & 1.73 & 0.97 \\
\hline \multirow[t]{2}{*}{14} & $1.00(81)$ & 2.77 & 1.70 & 0.97 \\
\hline & & (19) & & \\
\hline \multirow[t]{2}{*}{18} & $1.17(84)$ & 2.53 & 1.57 & 0.97 \\
\hline & & (16) & & \\
\hline
\end{tabular}

In water medium, due to poor solubility of PM-PB and PM-TAB there may be presence of small aggregates of the molecules along with the monomeric forms. This may be responsible for the biexponential decay of PM-PB and PM-TAB in water with a shorter and longer lifetime component. The monomeric form is expected to have a shorter lifetime value while the aggregated form to have longer lifetime. The amplitudes $\left(a_{1}, a_{2}\right)$ of the corresponding components, indicates the population of the respective components in their microenvironment. Amid a progressive formation of bile salt micellar aggregates on increasing $\mathrm{NaDC}$ concentration, there is an increase in amplitude and lifetime of the monomeric form. This may be due to the breaking of aggregated forms of PM-PB and PM-TAB in presence of bile salt and corresponding incorporation of the monomeric forms in to the NaDC micellar aggregates. In other words, there is an increase in solubility of PM-PB and PM-TAB with NaDC concentration. As compared to PM-TAB the variation is more prominent in case of PM-PB.

\section{Conclusions}

The synthesis and characterization of pyrene derivatives, $\mathrm{N}$-(1-Pyrenoylmethyl)pyridinium bromide (PM$\mathrm{PB}$ ) and $\mathrm{N}$-(1-Pyrenoylmethyl)-N,N,N-triethylammonium (PM-TAB) were discussed. With increase in solvent polarity from heptane to water, there is a negligible red shift of $\sim 10 \mathrm{~nm}$ in the spectral profile of 
PM-PB. However, PM-TAB shows a reasonable red shift of $\sim 50 \mathrm{~nm}$ in its emission spectral profile. The Stokes shift of PM-PB and PM-TAB fits well with ET (30) scale of solvent polarity. The steady state fluorescence intensity and dynamic fluorescence lifetime of these derivatives were found to be sensitive to the NaDC micellar aggregates. Present study provides a basic photophysical understanding of PM-PB and $P M-T A B$ in the absence and presence of physiologically important bile salts.

\section{Declarations}

\section{ACKNOWLEDGMENTS:}

The authors thank Prof. K. K. Balasubramanian and Prof A. K. Mishra of IIT Madras, India, for their valuable discussion and help in collecting fluorescence lifetime data.

\section{AUTHOR DECLARATIONS:}

Funding There are currently no funding sources.

Conflicts of interest There are no conflicts to declare.

Ethical Approval All authors declare Ethics approval.

Consent to Participate All authors Consent to participate.

Consent for Publication All authors Consent for publication.

Data Availability All the data that have been referred to in the manuscript without being included have been given in the Supporting Information. Figures showing emission spectra of PM-PB and PM-TAB in solvents of different polarity (Fig. S1), emission spectra of PM-PB and PM-TAB with increasing NaDC concentration from 0 to $18 \mathrm{mM}$ (Fig. S2) are available in the supporting information.

Code Availability Not applicable.

Authors' contributions Synthesis, chemical characterization has been done by MS, SD, and supervised by SKR, and KV. Photophysical studies has been done by MS, MM, and supervised by MM. All authors read and approved the final manuscript.

\section{References}

1. Niko Y, Hiroshige Y, Kawauchi S, Konishi G (2012) Tetrahedron, 68, 6177-6185 and reference their in

2. Subuddhi U, Haldar S, Sankararaman S, Mishra AK (2006) Photochemical and Photobiological Sciences 5:459-466

3. Subuddhi U, Haldar S, Sankararaman S, Mishra AK (2008) J Photochem Photobiol A 200:381-387

4. Sarkar I, Hemamalini A, Das TM, Mishra AK (2016) RSC Adv 6:27933-27943 
5. Milosavljevic BH, Thomas JK (1988) J Phys Chem 92:2997-3001

6. Su FK, Liau GF, Hong JL (2007) J Polym Sci Part B Polym Phys 45:920-929

7. Miller E, Styczynska DJ (2009) Spectrochimica Acta Part A 72:312-321

8. Coleman R (1987) Bile salts and biliary lipids. Kenneth S. Dodgson memorial symposium, Biochem. Soc. Trans., 15, S68-S89

9. Notter RH (2000) Lung Surfactants: Basic Science and Clinical Applications. Marcel Dekker Inc., New York

10. Erlinger S (1987) Physiology of Bile Secretion and Enterohepatic Circulation in Physiology of the Gastrointestinal Tract. Raven Press, New York

11. Mohapatra M, Mishra AK (2010) J Phys Chem B 114:14934-14940

12. Mohapatra M, Mishra AK (2011) Langmuir 27,13461-13467

13. Mohapatra M, Mishra AK (2013) Langmuir 29,11396-11404

14. Carey MC, Small DM (1972) Arch Intern Med 130:506

15. Gouin S, Zhu XX (1988) Langmuir, 14,4025

16. Ranajit P, Mathew MK, Narayanan R, Balaram P (1979) Chem Phys Lipids 25:345

17. Nakashima T, Anno T, Kanda H, Sato Y, Kuroi T, Fujji H, Nagadome S, Sugihara G (2002) Colloids Surf B 24:103

18. Subuddhi U, Mishra AK (2007) Colloids Surf B 57:102-107

19. Reis S, Moutinho CG, Matos C, de Castro B, Gameiro P, Lima JLFC (2004) Anal Biochem 334:117126

20. Abraham MH, Taft RW (1983) J Org Chem 48:2877-2887

21. Tripathi AK, Mohapatra M, Mishra AK (2015) Phys Chem Chem Phys 17:29985

22. Lorraine M, Deck, Daub GHOrg. Chem, 19873, 48,3577-3580

23. Avijit Jana S, Atta SK, Sarkar ND, Pradeep Singh (2010) Tetrahedron 66:9798-9807

24. Anuradha M (2007) Swati. Colloid Polym Sci 285:397-404

25. Jacques J, Marquet A (1973) Org Synthesis 53:111

26. Nynke M, van Spijker AM, Cornelisse J (1990) J. Org. Chem., 55, 756-758

27. Gridin VV, Kim TK, Bekkerman A, Bulatov V, Jung K-H, chechtera I (2003) Eur J Mass Spectrosc 9:187 28. Songjie Yu S, Liu Yu, Lan B Van (2015) J Am Chem Soc 137:1623-1631XingweiLi

29. Marcus Y (1991) J Solut Chem 20:929-944

\section{Figures}


(A)

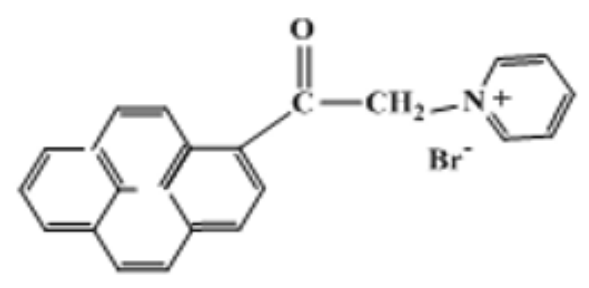

N-(1-Pyrenoylmethyl)pyridinium bromide
(B)

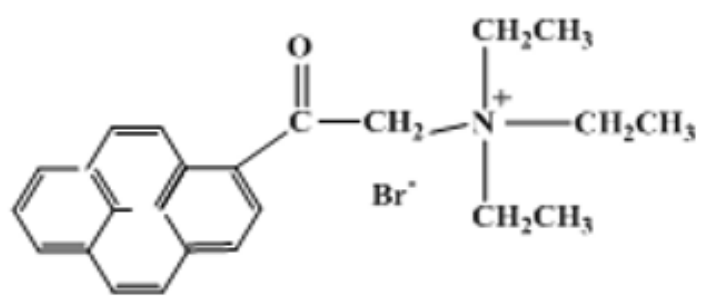

N-(1-Pyrenoylmethyl)-N,N,N-triethylammonium bromide

\section{Figure 1}

Molecular structures of (A) N-(1-Pyrenoylmethyl)pyridinium bromide (PM-PB) and (B) N-(1Pyrenoylmethyl)-N,N,N-triethylammonium bromide (PM-TAB).
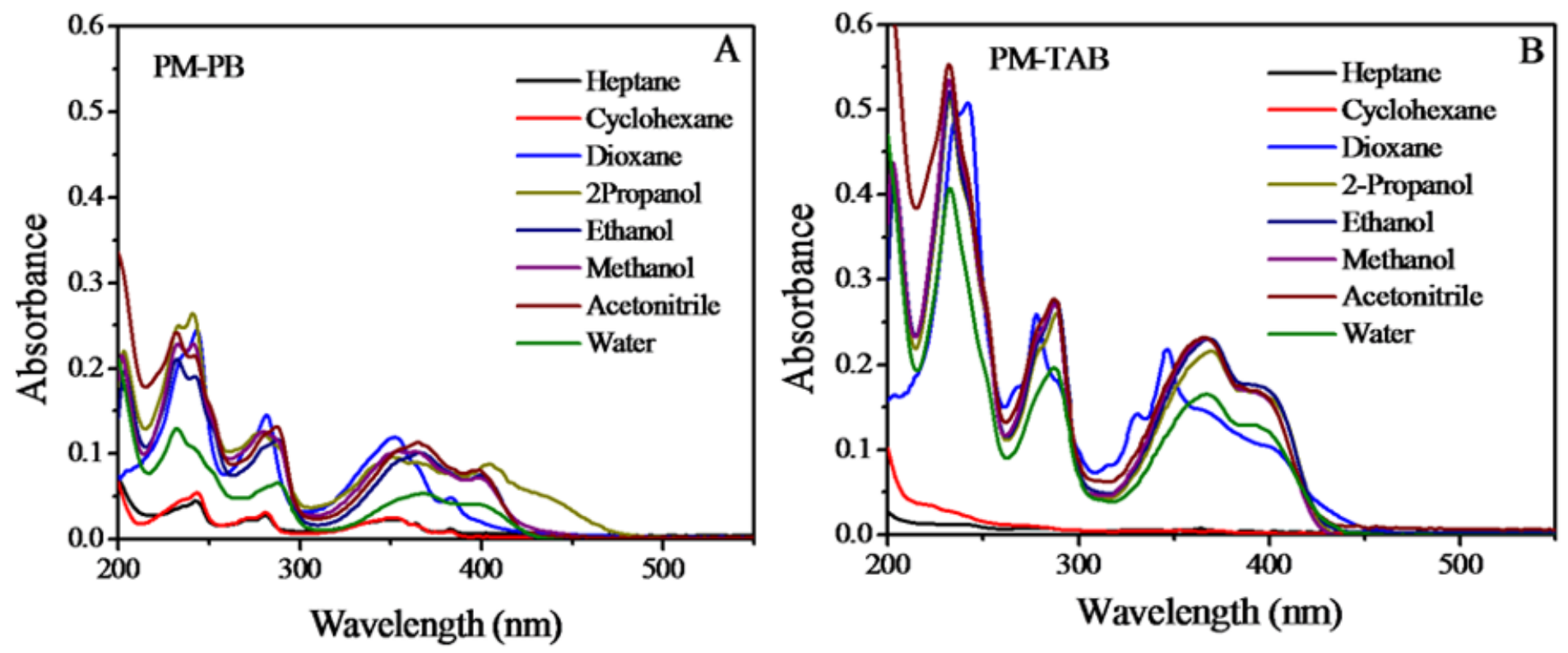

Figure 2

UV-Vis absorption spectra of (a) PM-PB and (b) PM-TAB in solvents of different polarity. (Concentration of $\mathrm{PM}-\mathrm{PB}$ and $\left.\mathrm{PM}-\mathrm{TAB}=5 \times 10^{-5} \mathrm{M}\right)$. 

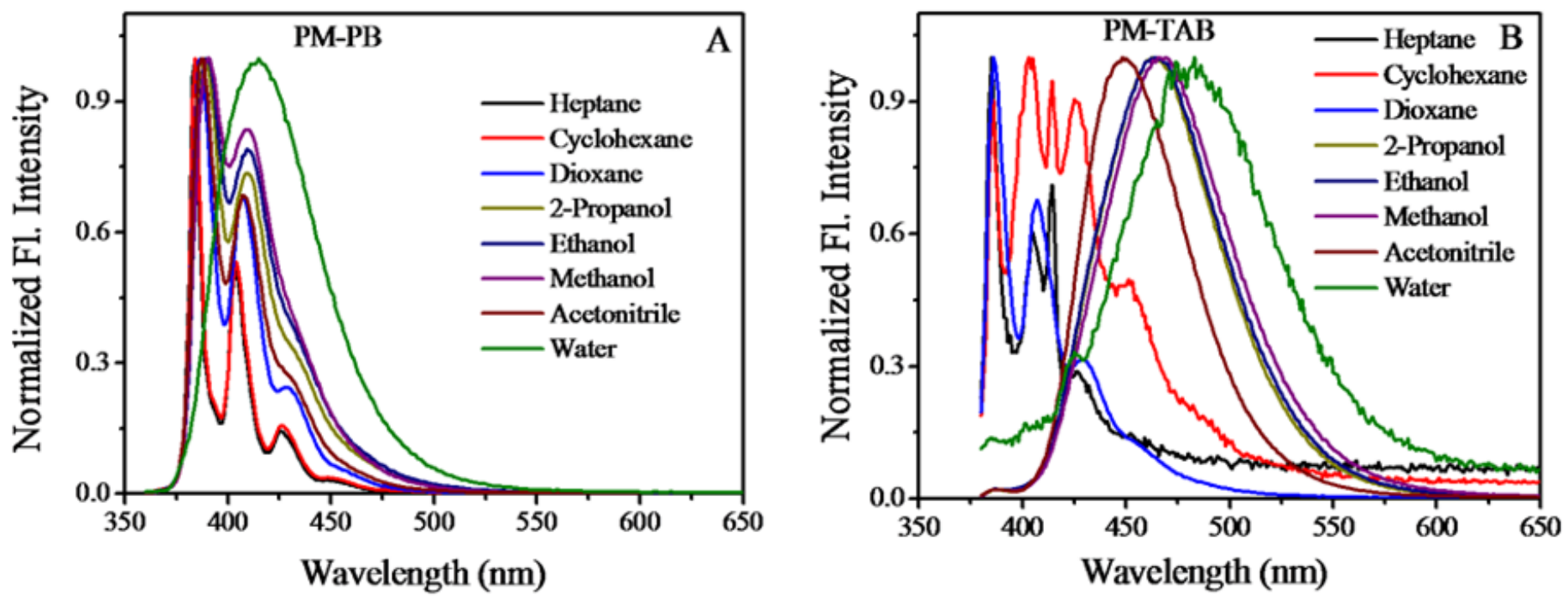

Figure 3

Normalized emission spectra of (A) PM-PB and (B) PM-TAB in solvents of different polarity. (Concentration of PM-PB and PM-TAB $=5 \times 10^{-5} \mathrm{M}, \lambda_{\mathrm{ex}}=370 \mathrm{~nm}$ ).
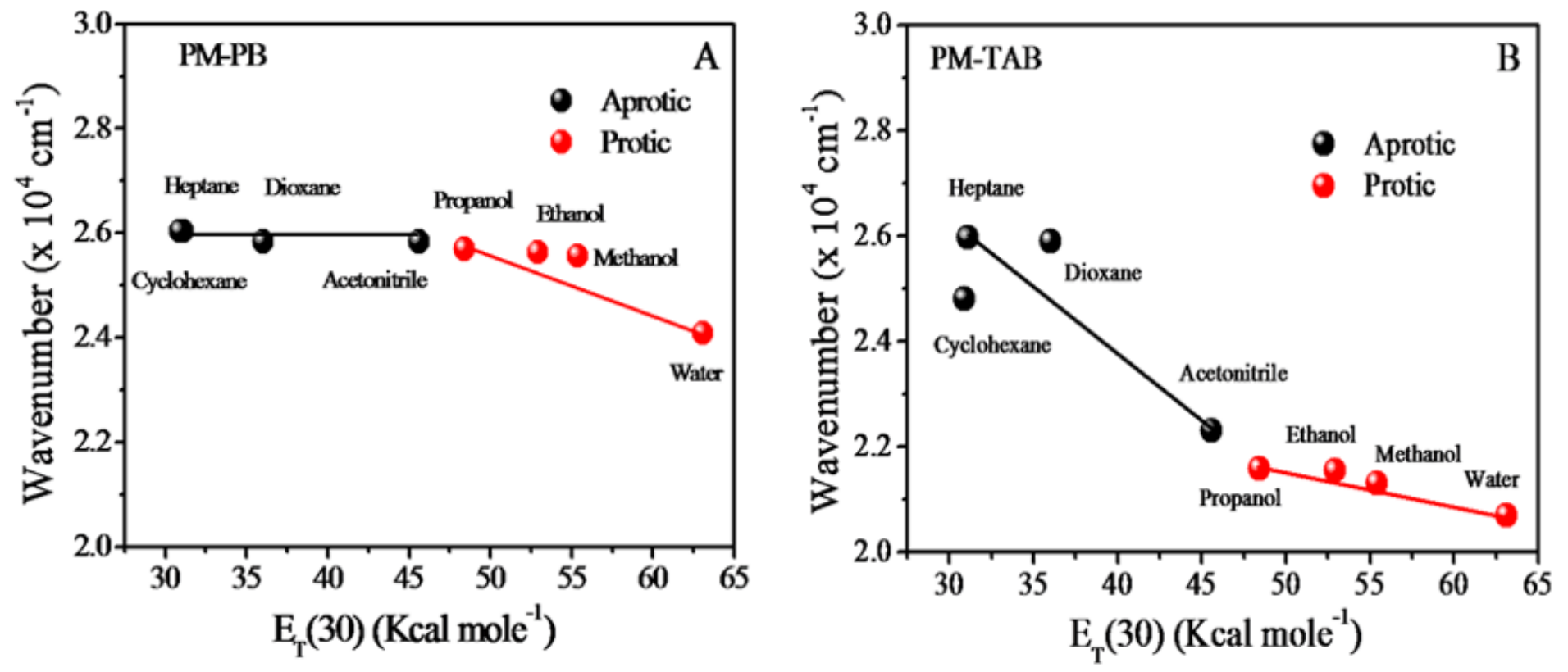

Figure 4

ET(30) plot for (A) PM-PB and (B) PM-TAB. 

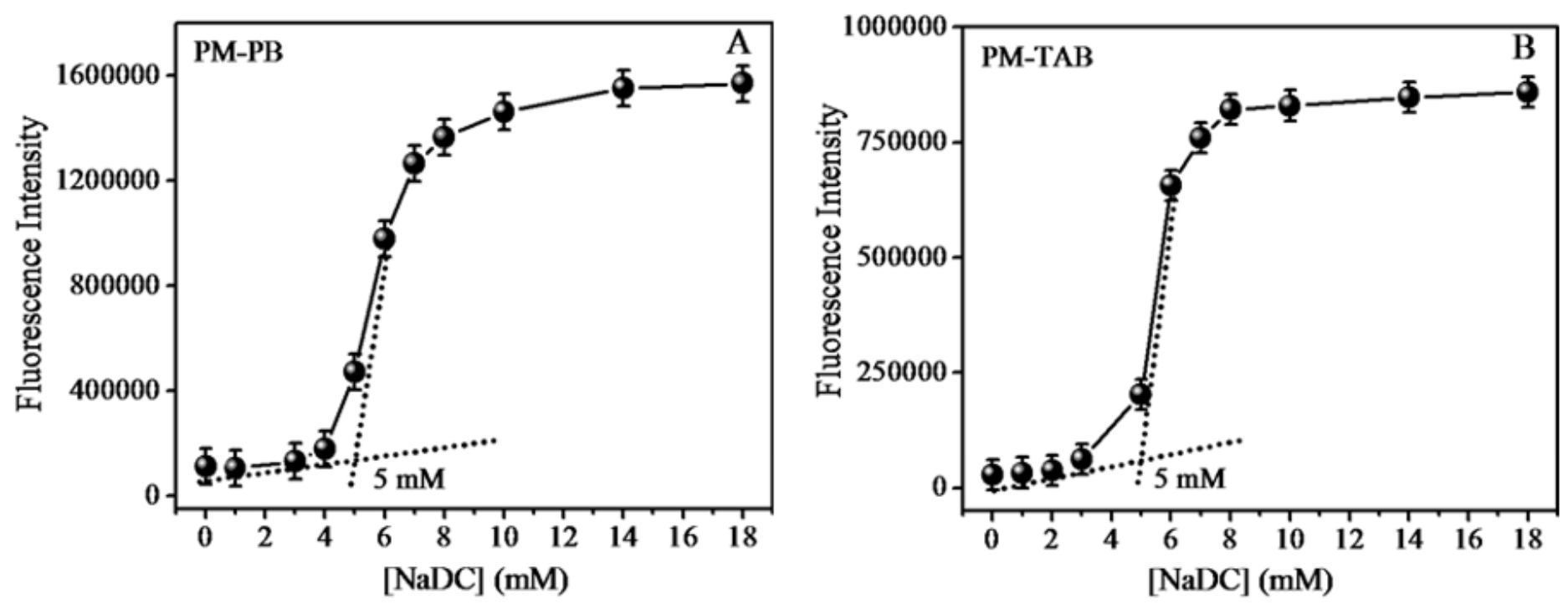

Figure 5

Plots of variation in steady state fluorescence intensity of (A) PM-PB and (B) PM-TAB with increasing $\mathrm{NaDC}$ concentration from 0to $18 \mathrm{mM}$. (Concentration of PM-PB and PM-TAB $=5 \times 10^{-5} \mathrm{M}, \lambda_{\mathrm{ex}}=370 \mathrm{~nm}$ ).
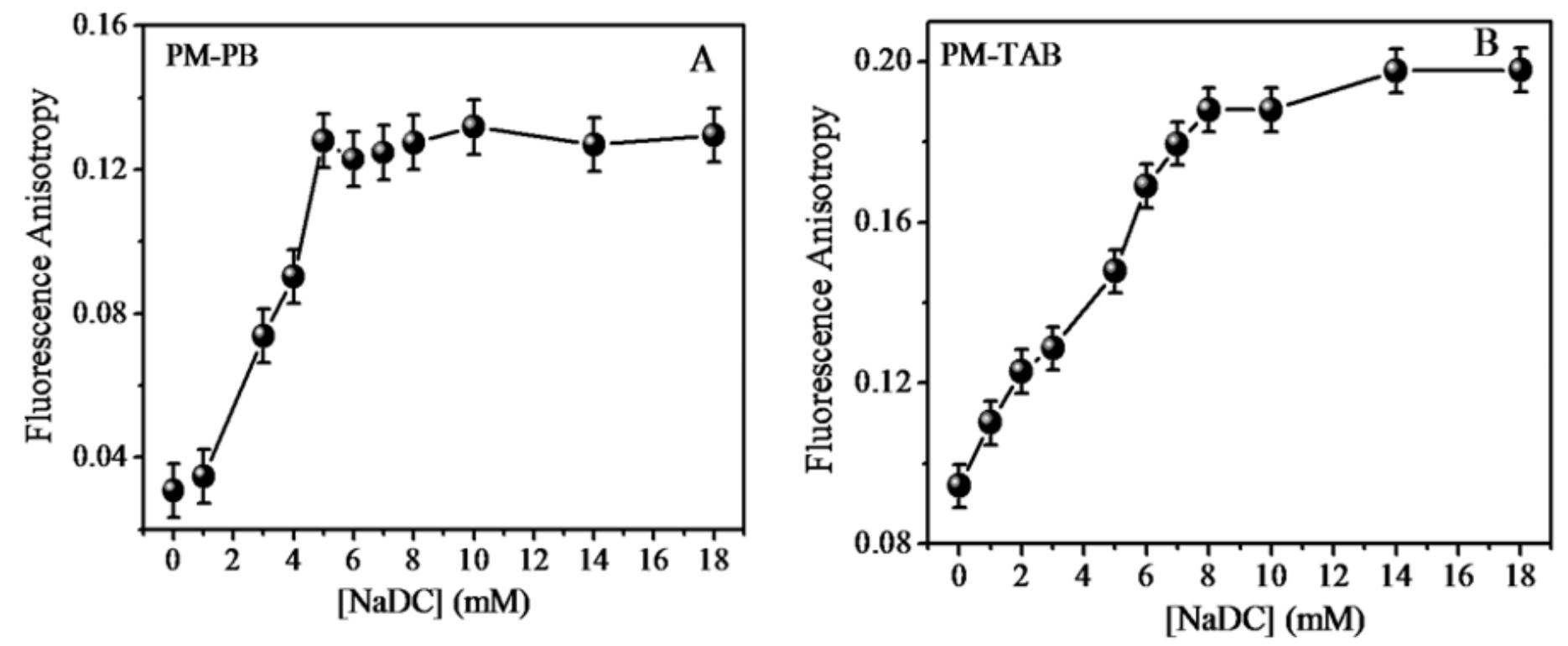

Figure 6

Plots of variation in steady state fluorescence anisotropy of (A) PM-PB and (B) PM-TAB with increasing $\mathrm{NaDC}$ concentration from 0 to $18 \mathrm{mM}$. 

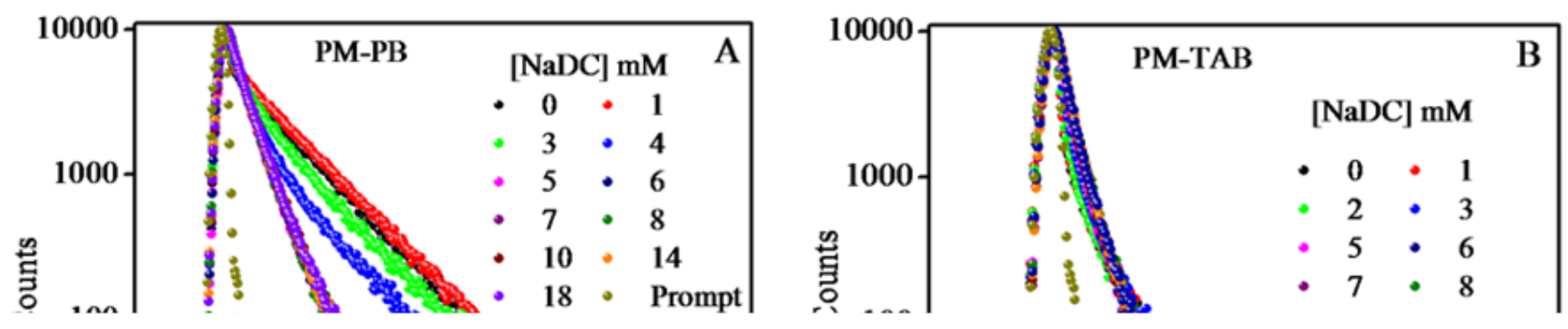

Figure 7

Decay profile of (A) PM-PB and (B) PM-TAB with increasing NADC concentration from 0 to $18 \mathrm{mM}$. $\left(\lambda_{\mathrm{ex}}=\right.$ $\left.370 \mathrm{~nm}, \lambda_{\mathrm{em}}=450 \mathrm{~nm}\right)$

\section{Supplementary Files}

This is a list of supplementary files associated with this preprint. Click to download.

- Supporting.docx 